IdeAs

Idées d'Amériques

$2 \mid 2012$

Universités d'Amériques et construction des savoirs

\title{
Les universités américaines dans les palmarès mondiaux
}

Hervé Théry

\section{(2) OpenEdition}

1 Journals

Édition électronique

URL : https://journals.openedition.org/ideas/255

DOI : $10.4000 /$ ideas.255

ISSN : 1950-570

Éditeur

Institut des Amériques

Référence électronique

Hervé Théry, "Les universités américaines dans les palmarès mondiaux », IdeAs [En ligne], 2 | 2012, mis en ligne le 22 juin 2012, consulté le 18 octobre 2022. URL : http://journals.openedition.org/ideas/ 255 ; DOI : https://doi.org/10.4000/ideas.255

Ce document a été généré automatiquement le 18 octobre 2022

\section{(c) (i) () $९$}

Creative Commons - Attribution - Pas d'Utilisation Commerciale - Pas de Modification 4.0 International - CC BY-NC-ND 4.0

https://creativecommons.org/licenses/by-nc-nd/4.0/ 


\title{
Les universités américaines dans les palmarès mondiaux
}

\author{
Hervé Théry
}

1 Comment se situent les universités américaines dans les classements mondiaux d'Universités ? Outre la classification « de Shanghai », très critiquée, en fonction de ses présupposés et de ses limitations, d'autres palmarès ont surgi dans plusieurs pays du monde. Parmi ceux-ci, nous en avons choisi trois, établis par des institutions de trois pays (France, Grande-Bretagne et Espagne), sur des bases elles aussi différentes : celle produite par l'école des Mines de Paris, qui privilégie la réussite de ses anciens élèves dans le monde des affaires ; celle du Times Higher Education (britannique), qui analyse dans la mesure du possible - la qualité de la formation donnée aux étudiants ; et enfin celle du laboratoire Webometrics, du Consejo Superior de Investigaciones Científicas espagnol, qui mesure la qualité et la visibilité des sites Web des universités.

2 Ainsi pourra-t-on vérifier si, bien que construit selon des principes et avec des données différentes, ils aboutissent - ou non - à des cartes semblables. Un premier point mérite réflexion: pour les quatre classements retenus, la concentration des universités classées dans quelques régions du monde est telle qu'il a fallu ajouter au planisphère trois zooms détaillant la situation des États-Unis, de l'Europe et du Japon.

\section{Arwu, le « classement de Shanghai »}

Ce palmarès (dont le nom officiel est ARWU, Academic Ranking of World Universities) classe annuellement, depuis 2003, les principales universités mondiales en fonction de leurs « résultats de recherches » comme le disent très clairement ses auteurs, qui ont pour unique ambition de produire un classement "fondé sur des données internationalement comparables et que chacun pourrait vérifier ». Il analyse toutes les Universités dont les ex-étudiants ou enseignants ont reçu un prix Nobel, une médaille Fields, ont eu des articles publiés dans Nature ou Science, dont les chercheurs figurent parmi les plus cités, ainsi que celles qui ont un nombre significatif d'articles indexés par Science Citation Index-Expanded (SCIE) and Social Science Citation Index (SSCI). Soit au 
total un millier d'établissements, dont seuls les 500 premiers sont cités dans la liste publiée.

Tableau Les critères du classement Arwu

\begin{tabular}{|c|c|c|c|}
\hline Critères & Indicateur & Code & Poids \\
\hline $\begin{array}{l}\text { Qualité de } \\
\text { l'éducation }\end{array}$ & $\begin{array}{l}\text { Anciens élèves d'un établissement ayant reçu des médailles } \\
\text { Fields et des prix Nobel }\end{array}$ & Alumi & $10 \%$ \\
\hline \multirow{2}{*}{$\begin{array}{l}\text { Qualité du corps } \\
\text { enseignant }\end{array}$} & $\begin{array}{l}\text { Personnel d'un établissement ayant reçu des médailles } \\
\text { Fields et des prix Nobel }\end{array}$ & Award & $20 \%$ \\
\hline & Chercheurs les plus cités dans 21 champs disciplinaires & $\mathrm{HiCi}$ & $20 \%$ \\
\hline \multirow{2}{*}{$\begin{array}{l}\text { Résultats } \\
\text { recherches }\end{array}$} & Articles publiés dans Nature et Science $*$ & N\&S & $20 \%$ \\
\hline & $\begin{array}{l}\text { Articles répertoriés dans le Science Citation Index- } \\
\text { expanded, et le Social Science Citation Index }\end{array}$ & Pub & $20 \%$ \\
\hline $\begin{array}{l}\text { Taille } \\
\text { l'établissement }\end{array}$ & $\begin{array}{l}\text { Résultats des autres indices divisés par le nombre de } \\
\text { personnes employées par l'établissement }\end{array}$ & PCP & $10 \%$ \\
\hline Total & & & $100 \%$ \\
\hline
\end{tabular}

Source : Arwu 2008 * Pour des établissements spécialisés dans les sciences humaines et les sciences sociales telles comme la London School of Economics, N\&S n'est pas considéré, et le poids de N\&S est affecté à d'autres indicateurs (note des auteurs du classement).

4 On peut reporter ces résultats sur une carte : à l'intérieur des États-Unis se distingue nettement un bloc compact allant de la côte de l'Atlantique (Nouvelle-Angleterre) aux Grands Lacs, suivi par la Californie et dans une moindre mesure par le Texas et la Floride. En Europe la concentration des universités de rang mondial reconstitue pratiquement la « dorsale européenne ", du nord de l'Angleterre à la Lombardie. Enfin, avec le Japon l'on retrouve la troisième grande mégalopole mondiale, dont la géographie des grandes universités mondiales, vue par ce classement, confirme donc de façon éclatante la prédominance. Si des choix graphiques légèrement différents avaient joint le Canada aux États-Unis et l'Europe du Nord au reste de l'Union européenne, le corps principal du planisphère apparaîtrait encore plus vide, puisqu'en dehors des cas cités on n'y trouve que quelques établissements latino-américains (Mexico, São Paulo, Rio de Janeiro, Belo Horizonte, Porto Alegre, Santiago et Buenos Aires), des universités australiennes, néo-zélandaises, singapouriennes, chinoises, indiennes, et seulement trois universités africaines, toutes les trois situées en Afrique du Sud, au Cap, à Johannesbourg et à Durban.

5 Une des objections les plus fréquentes est de demander si l'on peut réellement mesurer les résultats des universités par des chiffres. À cela les auteurs répondent, non sans humour, que les professeurs le font constamment, en donnant des notes à leurs étudiants. Il faut seulement, dans le cas des universités, tout comme dans ceux des étudiants, se rappeler que l'on ne doit pas prendre ces notes comme des références absolues. 
6 La place des sciences sociales et des sciences humaines est manifestement sous-estimée dans ce classement, les auteurs le reconnaissent eux-mêmes, mais il avouent n'avoir pas pu trouver, pour ces domaines scientifiques, de critères correspondant aux exigences qu'ils s'étaient fixées, des mesures universellement reconnues comme valides et librement accessibles sur Internet. En particulier, le fait de ne pas pouvoir disposer d'un classement des livres, l'un des principaux moyens d'expression de ces sciences, a constitué un obstacle majeur à une prise en compte correcte des productions des universités spécialisées dans les sciences sociales.

7 Un autre facteur de distorsion est le fait que l'anglais est devenu, dans la plupart des domaines, la langue internationale et que les universitaires du monde anglophone sont beaucoup plus intégrés au circuit international que leurs homologues d'autres ensembles culturels. De ce fait, leur classement des universités a manifestement favorisé les pays dont l'anglais est la langue maternelle.

Figure $\mathrm{n}^{\circ}$ Classement global Arwu ou "de Shanghai"
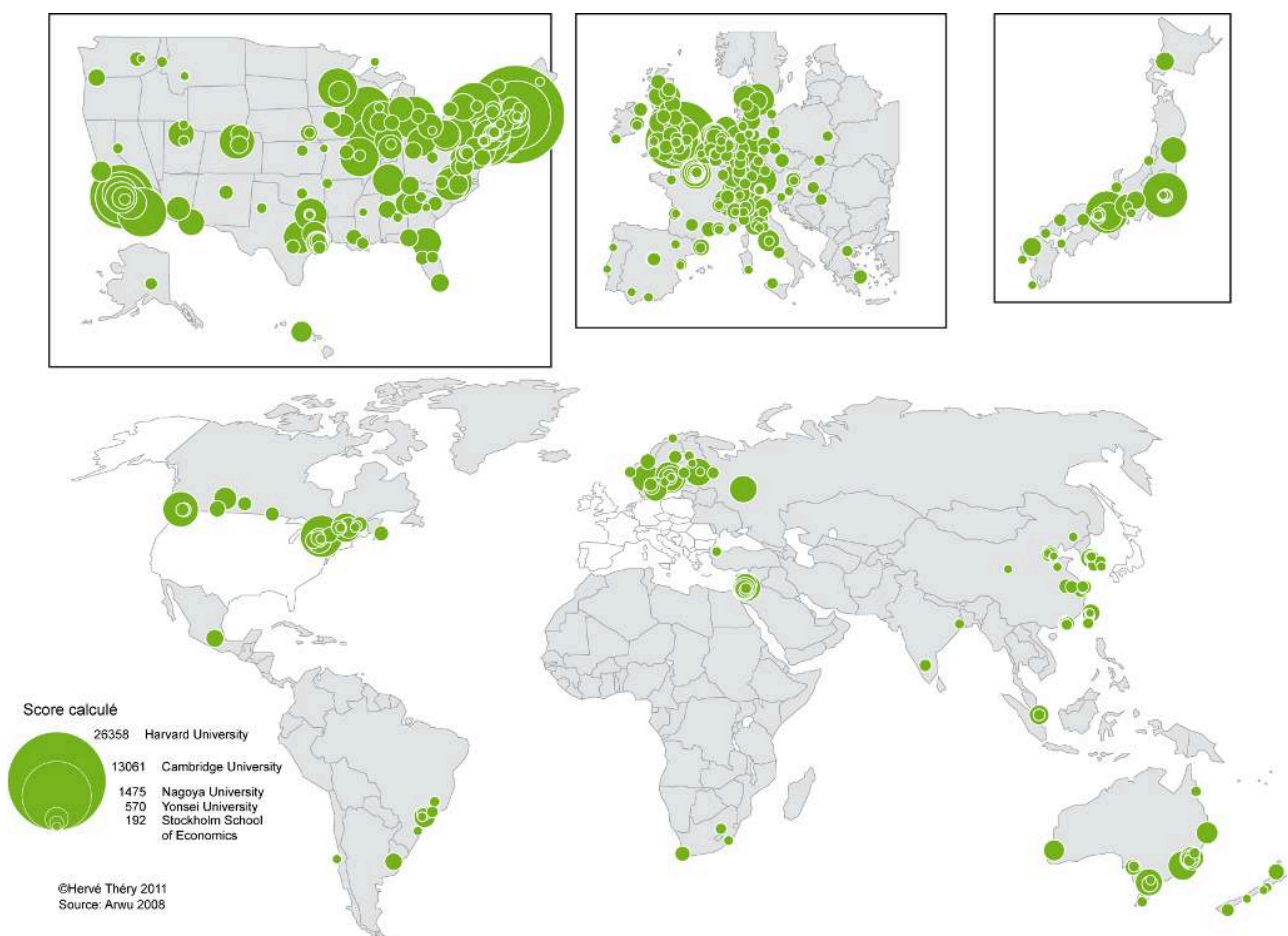

\section{Les autres classements}

En réponse à ce classement, où les «grandes écoles » françaises apparaissent peu, l'école des Mines de Paris (Mines ParisTech) a réalisé un «Classement international professionnel des établissements d'enseignement supérieur » établi à partir du nombre d'anciens étudiants figurant parmi les dirigeants des 500 plus grandes entreprises mondiales. Si quelques différences apparaissent dans les hiérarchies, comme la première place obtenue par Tokyo où l'apparition dans la tête du classement de HEC, de l'ENA ou de l'école Polytechnique, l'image d'ensemble qui apparaît sur la carte reste sensiblement la même. Tout au plus note-t-on que les universités californiennes y tiennent moins de place, que Paris et Tokyo obtiennent de meilleurs résultats et qu'un 
nombre un peu plus élevés d'universités chinoises et brésiliennes tirent leur épingle du jeu, en formant leurs cadres nationaux.

9 Souhaitant lui aussi se démarquer du classement de Shanghai, le classement établi par le Times Higher Education, journal britannique, référence reconnue dans les milieux universitaires, visait à prendre en compte les différentes fonctions de l'Université (au premier chef la formation des étudiants), et pas seulement les résultats de la recherche qui s'y fait. Cinq critères ont été retenus, qui vont du jugement porté par d'autres universitaires sur leurs concurrents (peer review) au jugement des employeurs, en passant par le nombre et la qualité des citations des travaux produits dans cette université, leur degré d'internationalisation et le taux d'encadrement des étudiants. La carte qui en est tirée confirme les indications données par le classement Arwu, la prédominance des trois mégalopoles, l'Américaine (élargie jusqu'aux Grands Lacs et complétée par la Sun Belt, de la Californie à la Floride), l'européenne et la japonaise, auxquelles il suffit d'ajouter quelques établissements au Canada, en Europe du Nord, en Chine et en Australie-Nouvelle Zélande pour obtenir l'essentiel de la géographie des grandes universités mondiales.

Le "Webometrics Ranking of World Universities" est une initiative du laboratoire Cybermetrics, un groupe de recherche appartenant au Consejo Superior de Investigaciones Científicas (CSIC), la plus grande institution publique de recherche en Espagne. Plus de 15000 universités ont été évaluées (contre 3000 par ARWU). Quatre indicateurs ont été obtenus : taille, visibilité, qualité des pages et contenu scientifique. La carte souligne une fois encore la prédominance des régions déjà mentionnées. Tout au plus peut-on dire que les petites et moyennes universités arrivent parfois à compenser, dans ce domaine, la disproportion des moyens dont elles disposent par rapport aux très grandes universités, leur site Internet pouvant être de qualité comparable sans de trop gros investissements.

\section{Un classement des classements?}

11 Au terme de l'analyse des quatre palmarès, un traitement statistique multivarié peut faire ressortir les différences entre eux et aider à construire une typologie des grands établissements d'enseignement supérieur dans le monde et à y situer celles des Amériques. Pour cela on a eu recours à une classification ascendante hiérarchique (CAH) des établissements (Universités et grandes écoles) présents dans les quatre classements, soit 96 au total : faire partie de cette courte liste est déjà un critère en soit, puisqu'il faut pour cela une reconnaissance quantifiée de sa production scientifique (Arwu), de sa capacité à produire des dirigeants de grands groupes économiques (Mines Paris Tech), de la qualité de son enseignement (Times Higher Education) et de sa présence sur Internet (Webometrics). Les résultats apparaissent sur la figure $n^{\circ} 2$, où les profils regroupent les établissements semblables au vu de ces critères. La CAH les regroupe en fonction de leur écart à la moyenne pour chaque variable, soit au dessus de la moyenne (barres à droite de l'axe vertical), soit au dessous de celle-ci (barres à gauche). 
Figure $n^{\circ}$ Les profils de la $\mathrm{CAH}$

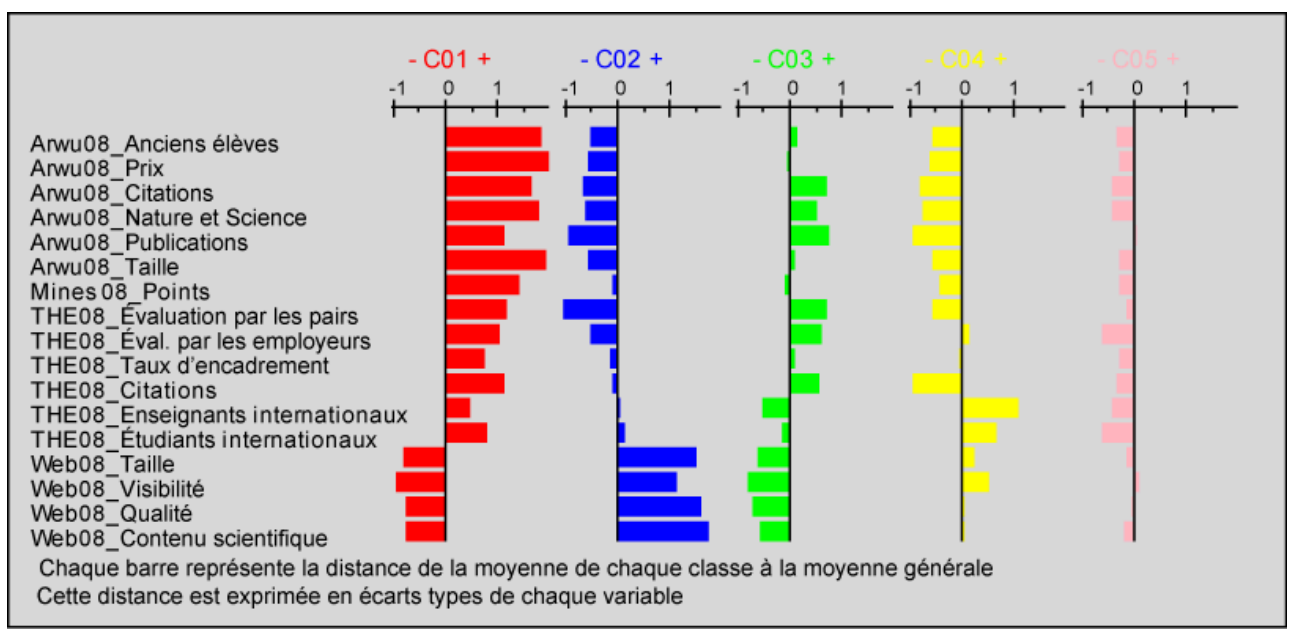

12 Le groupe $\mathbf{n}^{\circ} \mathbf{1}$ de la classification (cercles rouges) est visiblement celui des « meilleurs de la classe ", ceux qui ont des scores très supérieurs à la moyenne sur tous les critères, (à part leur présence sur le web, dont ils n'ont vraisemblablement pas tellement besoin). Ce sont principalement les universités de l'«Ivy league» de la Nouvelle Angleterre, plus Chicago, Oxford et Cambridge, Zurich (Institut Fédéral de Technologie) et Tokyo.

\section{Figure $n^{\circ}$ Carte de synthèse}

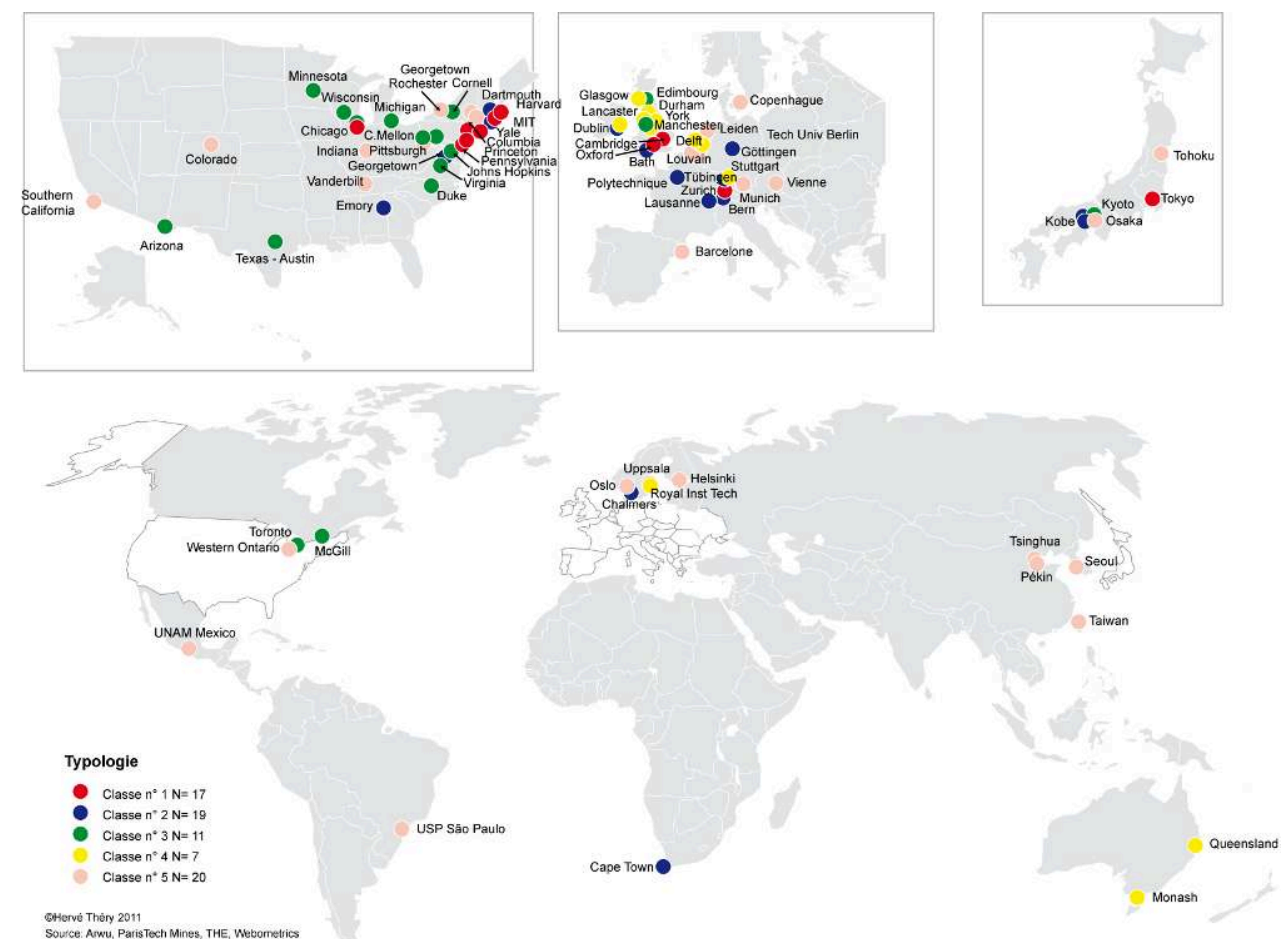

Le groupe $\mathbf{n}^{\circ} \mathbf{2}$ (cercles bleus) est celui qui se distingue principalement par la qualité et la fréquentation - de sa présence sur le Web. Il associe des universités un peu moins connues des États-Unis (Emory, Dartmouth, Georgetown) ou d'Europe (Bath, Göttingen, Tübingen, Chalmers), ainsi que l'École Polytechnique et l'Université de Cape Town, qui lui doivent respectivement la présence des seuls établissements français et africain. 
13 Le groupe $\mathbf{n}^{\circ} \mathbf{3}$ (cercles verts) se caractérise par des scores plus élevés que la moyenne pour les publications, notamment dans Nature et Science, une bonne évaluation par les pairs (et les employeurs), un grand nombre de citations de ses enseignants recensé dans les index reconnus, un profil plus "académique » et menant à de carrières moins brillantes - et rémunératrices - que le groupe $\mathrm{n}^{\circ} 1$. Il regroupe des universités du Middle West et du Sud des États-Unis, Mc Gill et Toronto, Kyoto, Manchester et Edimbourg.

14 Le groupe $\mathbf{n}^{\circ} \mathbf{4}$ (cercles jaunes) est le plus ouvert sur le monde, celui qui compte le plus d'étudiants et de professeurs étrangers, des sites abondants et très visités (une chose expliquant probablement l'autre): ces universités australiennes, hollandaises, irlandaises et surtout britanniques (Glasgow, Durham, York, Sheffield) ont su se rendre attractives par d'autres moyens que celles des États-Unis ou du Japon, dont on ne compte aucune dans ce groupe.

Le groupe $\mathbf{n}^{\circ} 5$ (cercles roses), finalement, est proche de la moyenne dans tous les domaines, il semble être, dans cette élite mondiale, celui des pays émergents (Chine, Brésil, Mexique), des régions périphériques - du point de vue universitaire - de l'Europe (Scandinavie, Espagne, Autriche, Belgique). Aux États-Unis il est formé d'établissements moins prestigieux que ceux du premier groupe, moins présents sur le web que le deuxième, qui publient moins que le troisième, moins international que le quatrième, mais doit qui d'être présent ici au fait qu'il fait tout de même figure honorable dans tous ces domaines. C'est là une belle performance alors que d'autres sont absents de cette short list faute de se qualifier dans l'un ou l'autre de ces champs de compétence : c'est notamment le cas de toute la Californie, à une exception près.

Ayant examiné les résultats des classifications qui se veulent les concurrentes de celles de Shanghai, on ne peut qu'être frappé par leur convergence, par le fait que les images que l'on peut construire en reportant - sans a priori - leurs résultats sur des cartes sont relativement semblables, à quelques détails près. Les images globales - les concentrations des «bons " établissements dans les trois mégalopoles, leur rareté ailleurs - sont très semblables dans les quatre cas, et l'analyse cartographique le fait ressortir de façon éclatante. Mais même dans ce contexte de domination des mégalopoles, des stratégies et des situations alternatives se font jour. À côté des "meilleurs de la classe», d'autres universités se distinguent par un profil plus " académique ", la qualité de leur présence sur le Web ou leur ouverture sur le monde. En outre, une nouvelle génération de régions et pays «émergents » se fraie un chemin jusqu'au groupe de tête, annonce - peut-être? - de futurs rééquilibrages.

\section{BIBLIOGRAPHIE}

Sources des données

Academic Ranking of World Universities, Institute of Higher Education, Shanghai Jiao Tong University, http://www.arwu.org/, consulté le 24/8/09. 
Lauréats de prix Nobel, http://nobelprize.org/nobel_prizes/, consulté le 24/8/09.

Médailles Fields, http://www.mathunion.org/general/prizes/fields/prizewinners/, consulté le 24/8/09.

Chercheurs les plus cités, http://www.isihighlycited.com, consulté le 24/8/09.

Articles édités dans Nature et Science, http://www.isiknowledge.com, consulté le 24/8/09.

Articles classés Science Citation Index-expanded et Social Science Citation Index, http://

www.isiknowledge.com, consulté le 24/8/09.

Ranking of World Universities in the Web, http://www.webometrics.info/, consulté le 24/8/09.

The Times Higher Education World University Ranking, http://www.timeshighereducation.co.uk/ hybrid.asp?typeCode=416\&pubCode=1\&navcode=137, consulté le 24/8/09.

Classement international professionnel des établissements d'enseignement supérieur, http:// www.mines-paristech.fr/Actualites/PR/, consulté le 24/8/09.

Fortune Global 500, http://money.cnn.com/magazines/fortune/global500/2009/ , consulté le 24/8/09.

\section{RÉSUMÉS}

Comment se situent les Universités américaines dans les classements mondiaux d'Universités, celui dit «de Shanghai » et les autres? Reporter leurs résultats sur une carte mondiale fait apparaître une géographie marquée dans les trois grandes mégalopoles, mais des stratégies alternatives semblent possibles.

How do the American universities stand in the world rankings of universities, the so-called "Shanghai" ranking and the others? Plotting results on a world map shows a marked geographical concentration in the main three megalopolises, but alternative strategies seem possible.

¿Cómo están las universidades americanas en el ranking mundial de universidades, el "de Shanghái" y los demás? Colocar los resultados en un mapa del mundo muestra una marcada concentración geográfica en las tres grandes megalópolis, pero estrategias alternativas parecen posibles.

\section{INDEX}

Mots-clés : Universités, classements, mégalopoles

Palabras claves : Universidades, clasificaciones, megalópolis.

Keywords : Universities, classifications, megalopolises

\section{AUTEUR}

\section{HERVÉ THÉRY}

Directeur de recherche au CNRS. Creda UMR2772 CNRS/Paris III Sorbonne Nouvelle. Professor visitante à l'Université de São Paulo (USP) hthery@aol.com 